\title{
Time-resolved Near-Edge X-ray Absorption Fine Structure (NEXAFS) Spectroscopy on photo-induced phase changes using a tabletop XUV Spectrometer
}

\author{
P. Grossmann ${ }^{1, a, b)}$, J. Norpoth ${ }^{2, b)}$, I. Rajkovic ${ }^{3, b)}$, L. Wegewitz ${ }^{4}$, \\ W. Maus-Firedrichs ${ }^{4}$, R. Moré ${ }^{3}$, S. Techert ${ }^{3}$, C. Jooss ${ }^{2}$ and Klaus Mann ${ }^{1}$ \\ ${ }^{1}$ Laser-Laboratorium Göttingen e.V., Hans-Adolf-Krebs-Weg 1, \\ D-37077 Göttingen, Germany \\ ${ }^{2}$ Institut für Materialphysik (IMP), Friedrich-Hund-Platz 1, D-37077 Göttingen, Germany \\ ${ }^{3}$ Max-Planck-Institut für biophysikalische Chemie, Am Faßberg 11, \\ D-37077 Göttingen, Germany \\ ${ }^{4}$ Institut für Physik und Physikalische Technologien (IPPT), Leibnizstraße 4, \\ D-38678 Clausthal-Zellerfeld, Germany
}

We present a tabletop XUV spectrometer based on a stable laser-driven X-ray source, making use of a gas-puff target. With this setup optical light pump / soft x-ray probe NEXAFS experiments with a temporal resolution of $\sim 230 \mathrm{ps}$ are feasible. Pump-Probe NEXAFS measurements were carried out in the "water window" region (2.28 nm - $4.36 \mathrm{~nm})$ on the manganite $\operatorname{Pr}_{0.7} \mathrm{Ca}_{0.3} \mathrm{MnO}_{3}$ (PCMO), investigating the diminutive changes of the oxygen k-edge that derive from an optically induced phase change. The results show the practicability of the tabletop XUV spectrometer on demanding investigations so far exclusively conducted at synchrotron radiation sources.

a) Electronic mail: peter.grossmann@1lg-ev.de

b) Authors contributed equally to the work 


\section{Introduction}

While the development of sources for future lithography at $13.5 \mathrm{~nm}$ advances rapidly ${ }^{1,2}$, applications beyond lithography are devised and improved in the EUV/XUV region. The development of laboratory-sized tabletop soft x-ray sources has led to an ever growing interest in setups for x-ray microscopy in the EUV and "water window" region $(2.28 \mathrm{~nm}$ $4.36 \mathrm{~nm})^{3-5}$ and absorption spectroscopy at the K-edges of low $Z$ elements ${ }^{6-9}$. Among others, the latter is a powerful tool for investigations of the near-edge $\mathrm{x}$-ray absorption fine structure (NEXAFS) for elemental and chemical analysis of samples. NEXAFS can be used to obtain element specific information, as it probes intermolecular bonds and distances, giving rise to changes in oxidation states.

Time resolved NEXAFS spectroscopy can be used to study transient changes in the electronic structures of photoinduced phase changes in elements and components, such as manganites like $\operatorname{Pr}_{0.7} \mathrm{Ca}_{0.3} \mathrm{MnO}_{3}$ (PCMO) ${ }^{10}$. Phase changes of various kinds of this and other colossal magnetoresistive (CMR) manganites have been of great interest as to understand the underlying mechanics leading to these interesting material behaviors ${ }^{11-13}$.

So far the use of the optical light pump / soft x-ray probe setup of table-top size has been demonstrated for nanosecond time-resolved x-ray diffraction experiments of $\mathrm{nm}$-sized periodicity ${ }^{14-16}$. Propagation of heat waves at nanosecond timescale on organic layered systems has been reported.

Here, we demonstrate the feasibility of conducting time-resolved NEXAFS measurements on the optical induced phase-change of PCMO with the aforementioned tabletop size XUV setup. The measurements on the transient changes of the electronic structure of the photoinduced phase change were carried out with a temporal resolution of $\sim 230 \mathrm{ps}$ and the change in absorption on the oxygen k-edge due to this phase change was determined 


\section{Setup}

The time-resolved NEXAFS experiments were performed in a vacuum system (FIG. 1 ), as the mean free path of soft x-ray radiation in air is less than $1 \mathrm{~mm}$. The $\mathrm{x}$-ray source is based on a laser-induced plasma, making use of a ps laser system (Ekspla, SL312P, $\lambda=1064 \mathrm{~nm}, \mathrm{f}=5 \mathrm{~Hz}, \mathrm{t}_{\text {pulse }}=170 \mathrm{ps}$ ) and a gas-puff target. The laser beam is focused into the source chamber and synchronized with a short krypton gas pulse created by a piezo driven fast gas valve (Proch-Trickl type ${ }^{17}$, backing pressure $10 \mathrm{bar}, \mathrm{t}_{\mathrm{open}}=900 \mu \mathrm{s}$ ). The light emitted from the hot and dense krypton plasma is filtered by an aluminum filter (thickness $200 \mathrm{~nm}$ ), eliminating out-of-band radiation, such as visible light. In the sample chamber, the PCMO sample is held by a cryostat (Oxford Instruments, Mikrostat, Temperature stability +/- $0.1 \mathrm{~K}$ ) and is cooled down to $100 \mathrm{~K}$ with liquid nitrogen.

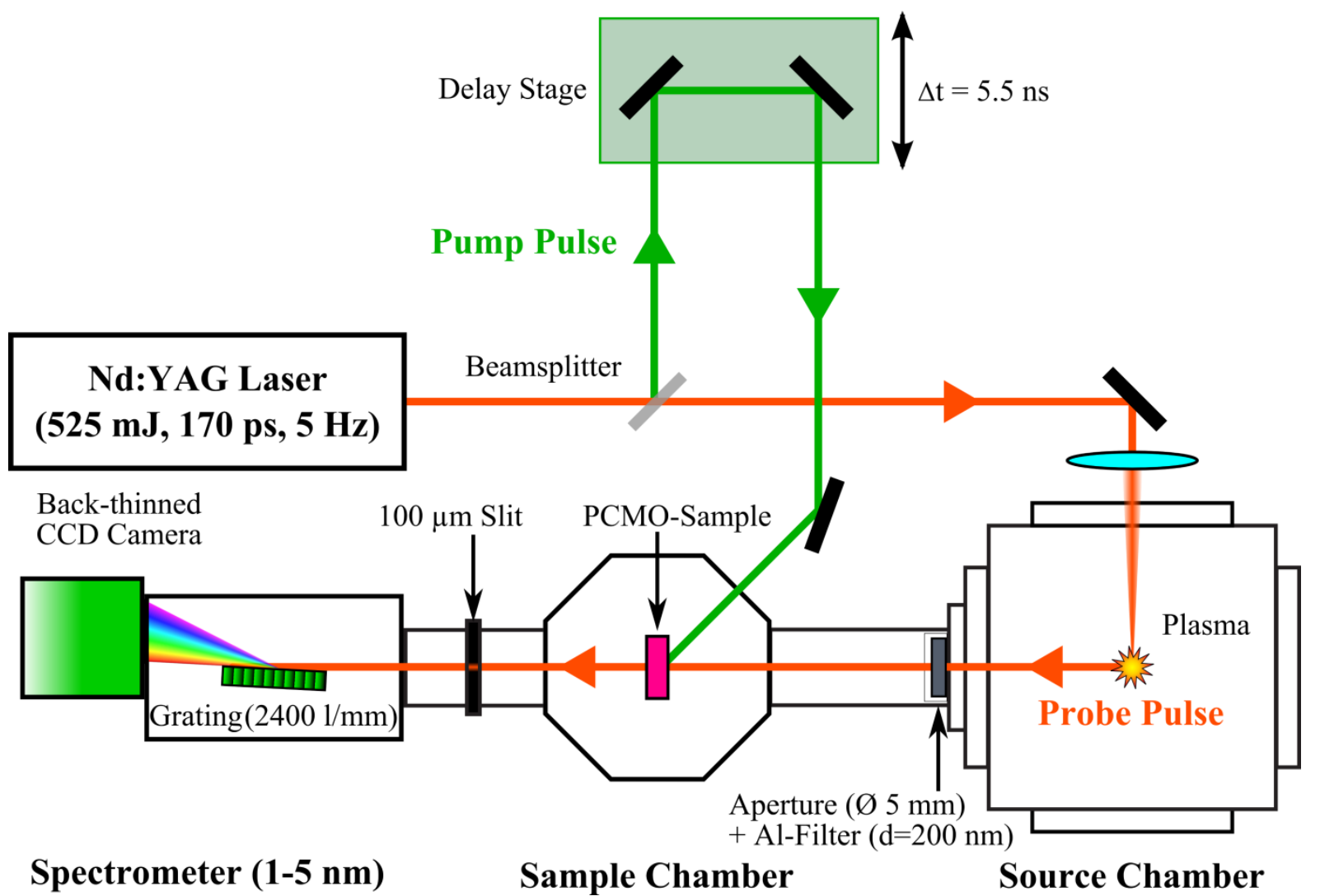

FIG. 1: Experimental Setup used for the pump-probe NEXAFS spectroscopy measurements. In addition to the shown components the sample-chamber was equipped with a cooling system based on liquid nitrogen. 
The window of the sample chamber for the pump pulse had an antireflective coating for IR radiation and apertures inside the sample chamber prevented stray light to pass into the spectrometer. The spectrometer is built up of an entrance slit $(\mathrm{d}=100 \mu \mathrm{m})$, an aberrationcorrected concave grating (Hitachi, 2400 grooves/mm, wavelength range $1-5 \mathrm{~nm}$ ) and a backside-illuminated CCD camera (Roper Scientific, 2048×512 Pixels, $13 \mu \mathrm{m} \times 13 \mu \mathrm{m}$ pixel size). With this setup the complete spectrum of the XUV source between $300 \mathrm{eV}$ and $1050 \mathrm{eV}$ is recorded within a single pulse.

The probe pulse was divided off the main pulse using a beamsplitter and its energy measured with a laser energy meter (Ophir Nova). The resulting energy of the pump beam was approx. $4 \%$ of the main pulse energy. The temporal overlap between the two pulses at the sample position was determined with an ultrafast photodiode, a fast oscilloscope (Tektronix, DPO 7254, $2.5 \mathrm{GHz}, 40 \mathrm{GS} / \mathrm{s}$ ) and the known path differences in the beam paths. The delay stage allowed for a delay of $-2.0 \mathrm{~ns}$ to $+3.5 \mathrm{~ns}$ between the pump pulse and the probe pulse at the sample surface.

\section{Time-resolved Measurements}

Optically pump/soft x-ray probe spectroscopy has been performed on a thin layer $(200 \mathrm{~nm})$ of PCMO. The layer was deposited onto a $\mathrm{Si}_{3} \mathrm{~N}_{4}$ sample window via pulsed laser deposition (PLD) at room-temperature at the IMP (Institut für Materialphysik, University of Göttingen). After deposition, the PCMO thin layer was annealed for 15 hours at $700^{\circ} \mathrm{C}$ under air and the crystal structure was verified via $\mathrm{x}$-ray diffraction (XRD) to be orthorhombic (space group Pbnm), as to be expected with the perovskite PCMO.

The PCMO thin film was investigated via $\mathrm{x}$-ray photoelectron spectroscopy (XPS), the $\mathrm{Mn}^{4+} / \mathrm{Mn}^{3+}$ ratio was verified and compared with literature, complying with the known ratio

for $\operatorname{Pr}_{0.7} \mathrm{Ca}_{0.3} \mathrm{MnO}_{3}$ (IPPT, Technical University, Clausthal) ${ }^{18-20}$. The measurements were performed at room temperature in an ultra high vacuum apparatus with a base pressure of $5 \times 10^{-11} \mathrm{hPa}{ }^{21}$ using a hemispherical analyzer (VSW HA100) and a commercial nonmonochromatic X-ray source (Specs RQ20/38C). For the measurement the $\mathrm{Al} \mathrm{K}$ line (photon energy $1486.7 \mathrm{eV}$ ) was used.

The sample was fixed on the cryostat via a copper frame, ensuring a uniform temperature over the sample size. The sample temperature was set to $100 \mathrm{~K}$ and the sample 
surface was positioned perpendicular to the probe-beam propagation. The pump pulse was aligned so that the angle of incidence was $45^{\circ}$ in respect to the sample surface, hitting the middle of the sample. The laser pulse energy was set to $240 \mathrm{~mJ}$, leading to a pump pulse of approx. $9 \mathrm{~mJ}$. The pump beam diameter was focused from $16 \mathrm{~mm}$ to $5 \mathrm{~mm}$, resulting in a fluence of approx. $30 \mathrm{~mJ} / \mathrm{cm}^{2}$, sufficient to optically induce the phase change of the PCMO ${ }^{22}$.

In order to measure the diminutive changes in the oxygen k-edge resulting from the photoinduced phase change, a high reproducibility over the course of the long measurement (1h and more) had to be maintained. This was achieved by using the laser in $1 \mathrm{~Hz}$ mode, as the pulse energy, i.e. the intensity of the plasma is dependent on the repetition rate of the laser. FIG. 2 shows the dependency of the plasma intensity on the repetition rate and time.

For this measurement the emission spectra of krypton plasmas have been accumulated over one minute and these spectra were integrated to obtain the total spectral intensity over one minute for the $1 \mathrm{~Hz}$ and $5 \mathrm{~Hz}$ repetition rate, respectively. For the $1 \mathrm{~Hz}$ mode the standard deviation is approx. $1.3 \%$ of the arithmetic average and only a slight trend downward can be observed. For the $5 \mathrm{~Hz}$ mode a drastic drop in intensity can be observed, leading to a standard deviation of approx. $4.7 \%$ of the arithmetic average. The trend of the intensity after 30 minutes is slightly modulated with a frequency of nearly 7 minutes. Further tests have shown that the drop in the intensity can be associated with the thermal load in the laser resonator and the low intensity modulation frequency of 7 minutes to the cooling cycle of the laser system. Consequently, the time-resolved NEXAFS measurements of PCMO were conducted with a repetition rate of $1 \mathrm{~Hz}$ to ensure a steady intensity of the XUV plasma source.

To further enhance the sensitivity during the measurement, for each timepoint the spectra were accumulated over 100 pulses and for each sample measurement one reference measurement was performed. The reference spectra were recorded with a time delay of $\Delta t=-2.0 \mathrm{~ns}$ and therefore well before the excitation of the sample through the pump pulse.

For transmission measurements the NEXAFS spectra are obtained through calculation of the optical thickness with the Lambert-Beer-Law

$$
O D_{\text {Sample }}=-\ln \left(\frac{I_{S}}{I_{0}}\right)
$$


where $O D$ is the optical thickness of the given sample, $I_{S}$ the transmitted (i.e. sample) and $I_{0}$ the reference intensities.
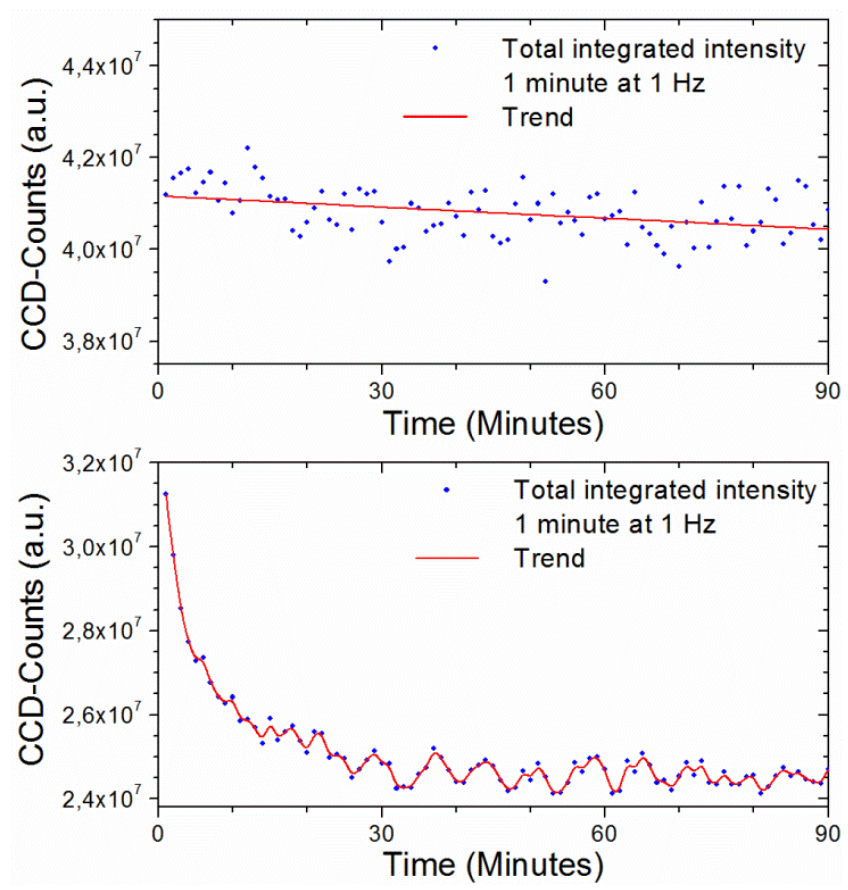

FIG. 2: Long-time stability of the plasma intensity while using the laser with $1 \mathrm{~Hz}$ (left) and $5 \mathrm{~Hz}$ (right). Each data point represents the total integrated intensity of the krypton spectra measured over 60 seconds.

The change in e.g. a bond length or oxidation state will result in a change in the intensity of an absorption edge and can be calculated by

$$
O D_{E}-O D_{R}=-\ln \left(\frac{I_{E}}{I_{R}}\right)
$$

with $O D_{E}$ being from the excited and $O D_{R}$ being from the reference sample measurement, respectively. This way, no moving of the sample was needed, as both spectra could be measured after passing through the sample, facilitating the measurement procedure. Too, the transmission of the same position of the sample was achieved. 


\section{Results and Discussion}

A static NEXAFS spectrum of PCMO is shown in FIG. 3, exhibiting absorption edges of every constituent element of PCMO. Synchronous monitoring of all absorption edges is therefore possible, as the whole spectral region is recorded at once. The nitrogen absorption edge derives from the $\mathrm{Si}_{3} \mathrm{~N}_{4}$ sample window.

Here, an investigation of the absorption changes in the oxygen K-edge (FIG. 3, inset) originating from the photoinduced phase change will be carried out. The peak at $\sim 530 \mathrm{eV}$ is of interest and arises from covalent hybridization between the oxygen $2 p$ orbital and the manganese $3 \mathrm{~d}$ orbital and therefore holds the information that control the conduction changes in $\operatorname{Pr}_{0.7} \mathrm{Ca}_{0.3} \mathrm{MnO}_{3}{ }^{10}$.

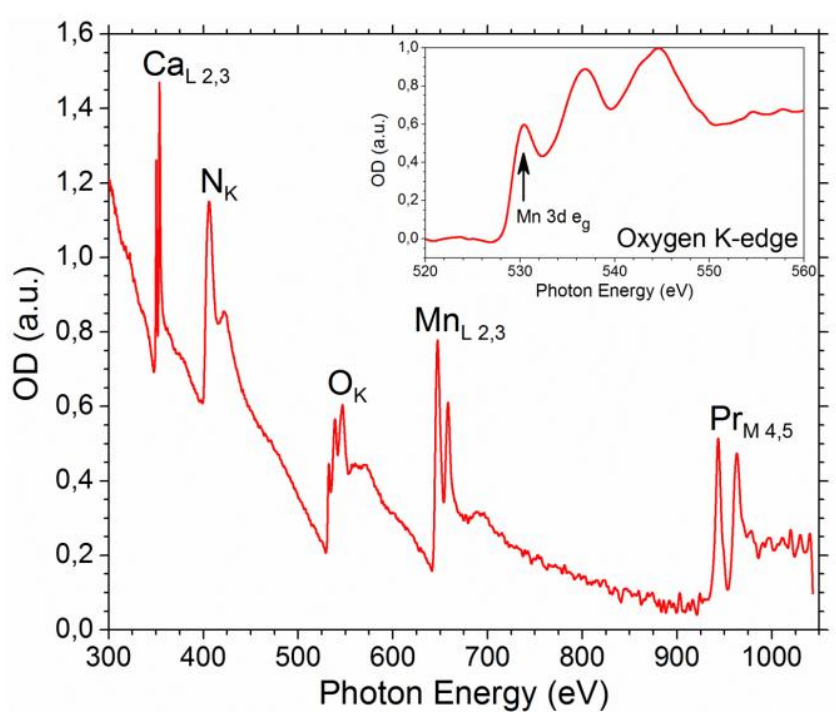

FIG. 3: NEXAFS-Spectrum of $\mathrm{Pr}_{0.7} \mathrm{Ca}_{0.3} \mathrm{MnO}_{3}(\mathrm{PCMO})$ in the water-window region and the section of the oxygen absorption K-edge (inset). As can be seen, each element of PCMO has an absorption-edge in the water-window, allowing for spectral investigations on every one of them. The nitrogen $\mathrm{K}$-edge derives from the $\mathrm{Si}_{3} \mathrm{~N}_{4}$ sample window.

To illustrate the principle of plasma based soft x-ray time-resolved spectroscopy, in FIG. 4 two NEXAFS spectra of distinguished edge features before and at the illumination are shown. The temporal evolution of the change in absorption at $\sim 530 \mathrm{eV}$ due to the photoinduced phase change is shown in FIG. 5. 


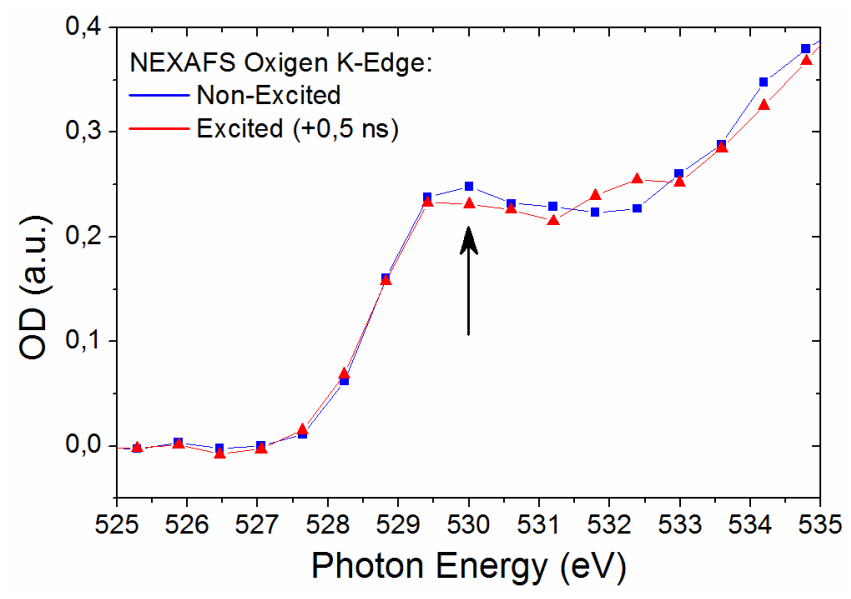

FIG. 4: Static NEXAFS spectra of the oxygen k-edge of non-excited PCMO (blue) and excited PCMO (red).

Before time zero between the pump and probe pulse there is basically no change in absorption. After time zero the absorption drops, exhibiting a time constant of $\tau=230 \mathrm{ps,}$ which is the temporal resolution-limit of the setup. Although being fairly diminutive in nature ( $3.5 \%$ change) the differences in absorption following the photo induced phase-change can be observed. This allows for further time resolved investigations on PCMO and other samples, that exhibit equally small or greater changes in absorption after excitation.

After approximately $1 \mathrm{~ns}$, the sample relaxes back into the unexcited state.

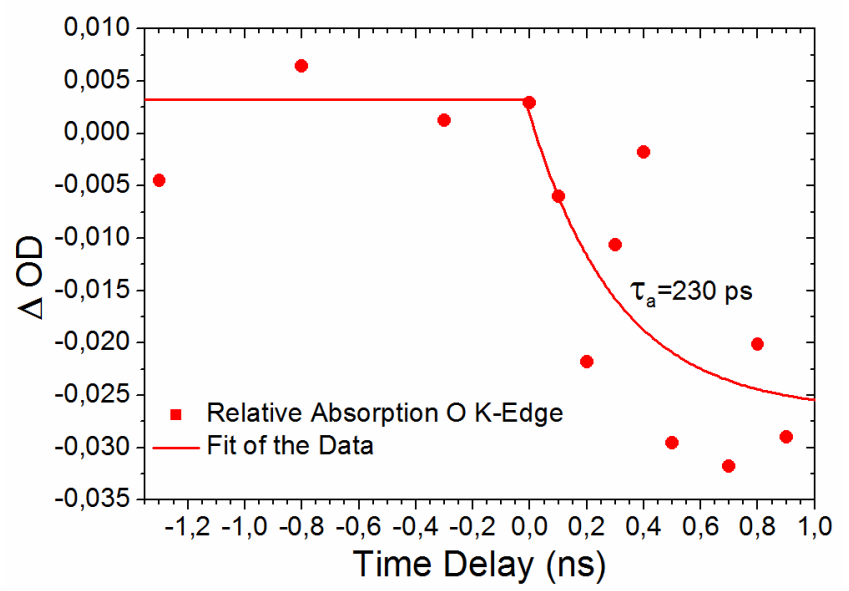

FIG. 5: Relative change in the absorption of the oxygen k-edge as a function of the pulse delay at $530 \mathrm{eV}$ due to the photoinduced phase change. The solid line between time zero and $0.7 \mathrm{~ns}$ is an exponential fit of the data points, which yields the temporal resolution of the setup. 


\section{V.Conclusion}

Recapitulatory, a tabletop XUV spectrometer based on a ps-laser induced plasma source is introduced, with which time-resolved NEXAFS spectroscopy can be performed, as shown with the case of the transient photoinduced phase change of the CMR manganite $\operatorname{Pr}_{0.7} \mathrm{Ca}_{0.3} \mathrm{MnO}_{3}$. Absorption changes at the oxygen k-edge of $3.5 \%$ were observed. This way, the feasibility of conducting time-resolved measurements on liquid nitrogen cooled samples with a temporal resolution or approximately 230 ps with a tabletop XUV spectrometer was demonstrated.

\section{Acknowledgments}

The authors would like to acknowledge the support by the DFG within the project SFB 602 "Komplexe Strukturen in kondensierter Materie von atomarer bis mesoskopischer Skala".

${ }^{1}$ B. Wu and A. Kumar, Journal of Vacuum Science \& Technology B: Microelectronics and Nanometer Structures 25, 1743-1761 (2007).

2 I.C.E. Turcu, C.M. Mann, S.W. Moon, and R. Allott, Microelectronic Engineering 35, 541-544 (1997).

${ }^{3}$ W. Chao, B.D. Harteneck, J.A. Liddle, E.H. Anderson, and D. Attwood, Nature 435, 1210-1213 (2005).

${ }^{4}$ H. Stollberg, P. Guttmann, P. a. C. Takman, and H.M. Hertz, Journal of Microscopy 225, 80-87 (2007).

${ }^{5}$ P. a. C. Takman, H. Stollberg, G.A. Johansson, A. Holmberg, M. Lindblom, and H.M. Hertz, Journal of Microscopy 226, 175-181 (2007).

${ }^{6}$ A. Braun, A. Kubatova, S. Wirick, and S.B. Mun, Journal of Electron Spectroscopy and Related Phenomena 170, 42-48 (2009).

${ }^{7}$ O. Dhez, H.W. Ade, and S.G. Urquhart, Journal of Electron Spectroscopy and Related Phenomena 128, 85-96 (2003).

${ }^{8}$ R.K. Hocking, S. DeBeer George, K.N. Raymond, K.O. Hodgson, B. Hedman, and E.I. Solomon, Journal of the American Chemical Society 132, 4006-4015 (2010). (2008)

${ }^{9}$ C. Peth, F. Barkusky, and K. Mann, Journal of Physics D: Applied Physics 41, 105202 
${ }^{10}$ M. Rini, Y. Zhu, S. Wall, R. Tobey, H. Ehrke, T. Garl, J.W. Freeland, Y. Tomioka, Y. Tokura, A. Cavalleri, and R. Schoenlein, Physical Review B 80, 1-5 (2009).

${ }^{11}$ M. Zimmermann, J. Hill, D. Gibbs, M. Blume, D. Casa, B. Keimer, Y. Murakami, Y. Tomioka, and Y. Tokura, Physical Review Letters 83, 4872-4875 (1999).

${ }^{12}$ M. Zimmermann, C. Nelson, Y.-J. Kim, J. Hill, D. Gibbs, H. Nakao, Y. Wakabayashi, Y. Murakami, Y. Tokura, Y. Tomioka, T. Arima, C.-C. Kao, D. Casa, C. Venkataraman, and T. Gog, Physical Review B 64, 1-9 (2001).

${ }^{13}$ Y. Tomioka, A. Asamitsu, H. Kuwahara, Y. Moritomo, and Y. Tokura, Physical Review B 53, 1689-1692 (1996).

${ }^{14}$ W. Quevedo, C. Peth, G. Busse, M. Scholz, K. Mann, and S. Techert, International Journal of Molecular Sciences 10, 4754-4771 (2009).

${ }^{15}$ W. Quevedo, C. Peth, G. Busse, K. Mann, and S. Techert, The Journal of Physical Chemistry B 114, 8593-8599 (2010).

${ }^{16}$ C. Peth, XUV-Laserplasmaquellen Für Die Absorptions-Spektroskopie Und Zeitaufgelöste Röntgenbeugung, Georg-August-Universität Göttingen, 2008.

${ }^{17}$ D. Proch and T. Trickl, Review of Scientific Instruments 60, 713-716 (1989).

${ }^{18}$ H. Nesbitt and D. Banerjee, American Mineralogist 83, 305-315 (1998).

${ }^{19}$ D.S. Kim, C.E. Lee, Y.H. Kim, and Y.T. Kim, Journal of Applied Physics 100, 093901 (2006).

${ }^{20}$ D. Hsu, A. Mani, C.H. Chuang, C.H. Chen, M.-T. Lin, and J.G. Lin, Journal of Applied Physics 105, 07 D505 (2009).

${ }^{21}$ M. Frerichs and F. Voigts, Applied Surface Science 253, 950-958 (2006).

${ }^{22}$ D. Polli, M. Rini, S. Wall, R.W. Schoenlein, Y. Tomioka, Y. Tokura, G. Cerullo, and A. Cavalleri, Nature Materials 6, 643-647 (2007). 